\title{
Valued Number And Set
}

Henry Garrett

Independent Researcher

DrHenryGarrett@gmail.com

Twitter's ID: @DrHenryGarrett | CB08PDK8J5G

\begin{abstract}
In this essay, the new notion concerning longest path is introduced. Longest path has a close relation with the notion of diameter in graph. The classes of graph are studied in the terms of having the vertex with longest path. Valued number is the number of edges belong to the longest path in the matter of vertex. For every vertex, there's a valued number and new notion of valued set is the generalization of valued number for the vertex when all vertices of the graphs are corresponded to a vertex which has the greater valued number. For any positive integer, there's one graph in that, there's vertex which its valued number is that. By deleting the vertices which don't belong to valued set, new notion of new graph is up. It's called valued graph. The comparison amid valued graph and initial graph is up, too.
\end{abstract}

Keywords: Valued Number, Valued Set, Longest Path, Valued Graph

AMS Subject Classification: 05C17, 05C22, 05E45, 05E14

\section{Outline Of The Background}

I'm going to refer to some books which are cited to the necessary and sufficient material which are covering the introduction and the preliminary of this outlet so look [Ref. [1], Ref. [2], Ref. [3], Ref. [4]] where Ref. [1] is about the textbook, Ref. [2] is common, Ref. [3] has good ideas and Ref. [4] is kind of disciplinary approaches in the good ways. Further references could be referred and could be addressed in Refs. [5-11].

\section{Definition And Its Clarification}

Definition 2.1. Let $\mathcal{G}=(\mathcal{V}, \mathcal{E})$ be a graph. For any given vertex $x$, the number $\sigma_{x}$ is called VALUED NUMBER if there's a vertex $y$ such that it has the longest $x y$-path where $x y$-path is a path from $x$ to $y$.

Definition 2.2. Let $\mathcal{G}=(\mathcal{V}, \mathcal{E})$ be a graph. A set $\mathcal{B} \subseteq \mathcal{V}$ is VALUED SET if for any of vertex $y$, there's a vertex $x$ belongs to $\mathcal{B}$, which there's a vertex such that its valued number is greater than, i.e., $\sigma_{x} \geq \sigma_{y}$. 


\section{Relationships And Its illustrations}

Theorem 3.1. Let $\mathcal{G}=(\mathcal{V}, \mathcal{E})$ be a complete graph. Thus every vertex is the valued set.

Theorem 3.2. Let $\mathcal{G}=(\mathcal{V}, \mathcal{E})$ be a complete graph. Thus the cardinality of valued set is one.

Theorem 3.3. Let $\mathcal{G}=(\mathcal{V}, \mathcal{E})$ be a complete bipartite graph. Thus the cardinality of valued set is two.

Theorem 3.4. Let $\mathcal{G}=(\mathcal{V}, \mathcal{E})$ be a complete bipartite graph. Thus every couple of vertices is the valued set where one vertex belongs to one part and another belongs to another part.

Theorem 3.5. Let $\mathcal{G}=(\mathcal{V}, \mathcal{E})$ be a path graph. Thus the cardinality of valued set is one

Theorem 3.6. Let $\mathcal{G}=(\mathcal{V}, \mathcal{E})$ be a path graph. Thus any of leaves is the valued set.

Theorem 3.7. Let $\mathcal{G}=(\mathcal{V}, \mathcal{E})$ be a cycle graph. Thus the cardinality of valued set is one.

Theorem 3.8. Let $\mathcal{G}=(\mathcal{V}, \mathcal{E})$ be a cycle graph. Thus any of vertices is the valued set.

Theorem 3.9. Let $\mathcal{G}=(\mathcal{V}, \mathcal{E})$ be a path graph. Thus the valued number of a vertex is $n-1$ if and only if it's leaf.

Theorem 3.10. Let $\mathcal{G}=(\mathcal{V}, \mathcal{E})$ be a cycle graph. Thus the valued number of any given vertex is $\left\lfloor\frac{n}{2}\right\rfloor$.

Theorem 3.11. Let $\mathcal{G}=(\mathcal{V}, \mathcal{E})$ be a cycle graph. Thus every vertex is the valued set.

Theorem 3.12. Let $\mathcal{G}=(\mathcal{V}, \mathcal{E})$ be a star graph. Thus every non-center vertex is valued set.

Theorem 3.13. Let $\mathcal{G}=(\mathcal{V}, \mathcal{E})$ be a star graph. Thus the valued number of every non-center vertex is two.

Theorem 3.14. Let $\mathcal{G}=(\mathcal{V}, \mathcal{E})$ be a wheel graph. Thus every vertex is valued set.

Theorem 3.15. Let $\mathcal{G}=(\mathcal{V}, \mathcal{E})$ be a wheel graph. Thus the valued number of every vertex is one.

\section{Results And Its Beyond}

Theorem 4.1. Let $\mathcal{G}=(\mathcal{V}, \mathcal{E})$ be a graph. Thus vertices which make diameter belong to valued set.

Theorem 4.2. Let $\mathcal{G}=(\mathcal{V}, \mathcal{E})$ be a graph. Thus every valued number is less than diameter.

Theorem 4.3. Let $\mathcal{G}=(\mathcal{V}, \mathcal{E})$ be a graph. Thus two given vertices which make diameter has the mutual valued number which is diameter.

Theorem 4.4. $n \in \mathbb{N}$. Thus there's a graph including a vertex which its valued number is $n$.

Theorem 4.5. Let $\mathcal{G}=(\mathcal{V}, \mathcal{E})$ be a graph. Thus the property of valued set is increasing monotone property. 
Definition 4.6. Let $\mathcal{G}=(\mathcal{V}, \mathcal{E})$ be a graph. For any set $\mathcal{B} \subseteq \mathcal{V}$ which is valued set, ${ }_{52}$ there's a graph which is obtained by $\mathcal{G}$ via deleting the vertices which don't belong to $\mathcal{B}$. ${ }_{53}$ It's called valued graph.

Theorem 4.7. Let $\mathcal{G}=(\mathcal{V}, \mathcal{E})$ be a complete graph. Thus the valued graph is $\mathcal{G}$.

Theorem 4.8. Let $\mathcal{G}=(\mathcal{V}, \mathcal{E})$ be a star graph. Thus the valued graph is $\mathcal{G}$ without its center.

Theorem 4.9. Let $\mathcal{G}=(\mathcal{V}, \mathcal{E})$ be a wheel graph. Thus the valued graph is $\mathcal{G}$.

Theorem 4.10. Let $\mathcal{G}=(\mathcal{V}, \mathcal{E})$ be a path graph. Thus the valued graph

- is disconnected;

- has two components;

- is from the order two;

- just includes leaves.

Theorem 4.11. Let $\mathcal{G}=(\mathcal{V}, \mathcal{E})$ be a cycle graph. Thus the valued graph is $\mathcal{G}$.

Theorem 4.12. Let $\mathcal{G}=(\mathcal{V}, \mathcal{E})$ be an union of two graphs. Thus

- the valued graph is an union of two valued graphs;

- the valued set is an union of two valued set;

- the initial valued number of any vertex is without change.

Theorem 4.13. Let $\mathcal{G}=(\mathcal{V}, \mathcal{E})$ be a complete bipartite graph. Thus the valued graph is

- $\mathcal{K}_{2}$;

- $\mathcal{P}_{2}$;

- connected;

- from order two where any two given vertices are from different parts.

Theorem 4.14. Let $\mathcal{G}=(\mathcal{V}, \mathcal{E})$ be a star graph. Thus the valued graph is

- center is deleted;

- $\mathcal{P}_{n-1}$;

- connected;

- from order $n-1$ where all vertices are non-center.

Theorem 4.15. Let $\mathcal{G}=(\mathcal{V}, \mathcal{E})$ be a join of one vertex and $\mathcal{P}_{n-1}$. Thus the valued graph is

- for any given vertex the valued number is two;

- $\mathcal{G}$;

- connected;

- from order $n$ where all vertices are. 
Theorem 4.16. Let $\mathcal{G}=(\mathcal{V}, \mathcal{E})$ be a join of one vertex and $\mathcal{K}_{n}$. Thus the valued graph ${ }_{85}$ is

- for any given vertex the valued number is one;

- $\mathcal{G}$;

- connected;

- from order $n$ where all vertices are.

Theorem 4.17. Let $x$ be a vertex. Consider join of $x$ and any given graph. Thus valued number of $x$ is one.

Theorem 4.18. Consider join of $\mathcal{P}_{1}$ and any given graph. Thus valued number of vertex of $\mathcal{P}_{1}$ is one.

\section{References}

1. R. Balakrishnan, K. Ranganathan, A Textbook of Graph Theory, New York, 2012.

2. Adrian Bondy, U.S.R Murty, Graph Theory, New York, 2008.

3. Michael Capobianco, and John C. Molluzzo, Examples and counterexamples in graph theory, New York, 1978.

4. Chris Godsil, and Gordon Royle, Algebraic Graph Theory, New York, 2001.

5. Henry Garrett, Big Sets Of Vertices, Preprints 2021, 2021060189 (doi: 10.20944/preprints202106.0189.v1).

6. Henry Garrett, Locating And Location Number, Preprints 2021, 2021060206 (doi: 10.20944/preprints202106.0206.v1).

7. Henry Garrett, Metric Dimensions Of Graphs, Preprints 2021, 2021060392 (doi: 10.20944/preprints202106.0392.v1).

8. Henry Garrett, New Graph Of Graph, Preprints 2021, 2021060323 (doi: 10.20944/preprints202106.0323.v1).

9. Henry Garrett, Numbers Based On Edges, Preprints 2021, 2021060315 (doi: 10.20944/preprints202106.0315.v1).

10. Henry Garrett, Matroid And Its Outlines, Preprints 2021, 2021060146 (doi: 10.20944/preprints202106.0146.v1).

11. Henry Garrett, Matroid And Its Relations, Preprints 2021, 2021060080 (doi: 10.20944/preprints202106.0080.v1). 